\title{
Is the life-world reduction sufficient in quantum physics? ${ }^{1}$
}

\author{
Michel Bitbol \\ Continental Philosophy Review, DOI 10.1007/s11007-020-09515-8, 2020 \\ This is a draft. The definitive version is available at: \\ Springer Link
}

\begin{abstract}
According to Husserl, the epochè (or suspension of judgment) must be left incomplete. It is to be performed step by step, thus defining various layers of "reduction". In phenomenology at least two such layers can be distinguished: the lifeworld reduction, and the transcendental reduction. Quantum physics was born from a particular variety of the life-world reduction: reduction to observables according to Heisenberg, and reduction to classical-like properties of experimental devices according to Bohr. But QBism has challenged this limited version of the phenomenological reduction advocated by the Copenhagen interpretation. QBists claim that quantum states are "expectations about experiences of pointer readings", rather than expectations about pointer positions. Their focus on lived experience, not just on macroscopic variables, is tantamount to performing the transcendental reduction instead of stopping at the relatively superficial layer of the life-world reduction. I will show that quantum physics indeed gives us several reasons to go the whole way down to the deepest variety of phenomenological reduction, may be even farther than the standard QBist view: not only reduction to experience, or to "pure consciousness", but also reduction to the "living present".
\end{abstract}

\section{Introduction}

Quantum mechanics was born from a quick and thorough ontological tabula rasa, between the years 1924 and 1926, just after the ontological patchwork that characterized the birth of quantum theory from 1900 to 1924. There were two versions of the tabula rasa: replacing an old ontology with a new one, or permanently suspending ontologies. De Broglie and Schrödinger proposed to substitute a new ontology that included their so-called "matter waves", for the old corpuscular ontology. Heisenberg was more radical when he introduced his matrix mechanics of 1925. He performed the well-known "reduction to observables", namely a reduction of the representational scaffolding of the new theory to the variables that can be directly measured in atoms: the frequencies and intensities of spectral lines. Heisenberg thereby suspended traditional ontologies without replacing them with anything. As for Bohr, he advocated a sort of middle way between replacing and suspending ontologies. On the one hand he held on to the idea of "quantum objects" that can be approached by complementary representations. Yet, on the other hand, he tended to reduce physics to

\footnotetext{
${ }^{1}$ This work was supported by the Agence Nationale pour la Recherche (ANR-16-CE91-0005-01).
} 
what can be handled in a laboratory or said in dialogues between scientists. This is what he implied by declaring that quantum theory is nothing else and nothing more than a "symbolism" to predict experimental phenomena.

In Bohr's own terms,

\begin{abstract}
"The quantum-mechanical formalism ... represents a purely symbolic scheme permitting only predictions, on lines of the correspondence principle, as to results obtainable under conditions specified by means of classical concepts." 2

"The appropriate physical interpretation of the symbolic quantummechanical formalism amounts only to predictions, of determinate or statistical character, pertaining to individual phenomena appearing under conditions defined by classical physical concepts." 3
\end{abstract}

But let's come back to Heisenberg's radical move. The word "reduction", often used to characterize Heisenberg's strong version of the ontological tabula rasa, is obviously reminiscent of the various phenomenological reductions. Heisenberg himself did not use this word in his pioneering paper of 1925 . However, he performed two gestures that have a strong phenomenological flavor. Firstly, he ruled out the clumsy compromise of the old quantum theory, in which quantum rules were associated to semi-classical pictures. And, secondly, he decided to: "...establish a theoretical quantum mechanics, analogous to classical mechanics, but in which only relations between observable quantities occur." ${ }_{4}$

Beyond the disputable use of the word "reduction", the two-steps structure of Heisenberg's reasoning irresistibly evokes the dynamics of phenomenological reduction. Heisenberg started with suspending his belief in former pictures or former ontologies. And he then redirected attention towards the epistemic acts of measurement and symbolization. These two steps closely correspond to the succession of: (i) a phenomenological epochè, and (ii) a reflective move towards the reduced domain.

\title{
Are epochè and reduction the same thing?
}

Before I develop this parallel in more details, let me give some precisions about the crucial method of phenomenology. It combines

\footnotetext{
2 Bohr (1987, p. 41)

${ }^{3}$ Bohr (1987, p. 64)

${ }^{4}$ Heisenberg (1925)
} 
epochè and reduction. But are epochè and reduction two distinct operations indeed, or just two names for a single one?

Many authors consider that there is no distinction to be drawn between the concepts of epochè and reduction in Husserl's phenomenology. They claim that "Husserl never succeeded in clarifying the relation between epochè and reduction", or, even worse, that any distinction is in vain since "The epochè and the phenomenological reduction, as epistemological instantiations, are synonymous" $"$.

Yet Husserl was unambiguous about the fact that epochè and reduction represent two distinct steps in the subtle methodological approach to phenomenological inquiry.

The epochè, to start with, is a phase of neutralization of our natural belief in the objects that are referred to by nouns in ordinary language. In Husserl's terms, "This universal depriving of acceptance, this 'inhibiting' or 'putting out of play' of all positions taken toward the objective world ... [is called the] "phenomenological Epochè'."7 But such depriving of acceptance is not tantamount to explicit refusal; such putting out of play is not tantamount to denial. We must not forget that Husserl was careful to avoid any confusion between his position and skepticism, from which he nevertheless borrowed the Greek word "epoche" : "I am not negating this world as though I were a Sophist; I am not doubting its factual being as though I were a skeptic; rather I am exercising the phenomenological epochè which shuts me off from any judgment about spatio-temporal factual being." "The external world of objective things is neither asserted nor negated, the natural ontological attitude is neither endorsed nor rejected, and such neutral stance is precisely the epochè.

But how far should we push this cultivated neutrality, this radical inhibition of our spontaneous tendency to believe in the independent existence of what we can perceive and manipulate? Should we seek a universal epochè, in which we would live beyond belief and doubt about everything, including our own ego $^{9}$ ? According to Husserl, phenomenology does not require us to go that far. In fact, phenomenology qua new science of the transcendental realm, would not even be possible if we did not set limits to the epochè. "With good

\footnotetext{
${ }^{5}$ Perniola (2011)

${ }^{6}$ Boehm (1965)

${ }^{7}$ Husserl $(1995$, p. 20)

${ }^{8}$ Husserl $(2016, \S 32)$

${ }^{9}$ Such radical epochè, leading to an a-subjective phenomenology, was advocated by Jan Patočka. See J. Patočka, "Epochè et réduction", in : Patočka (2002)
} 
reason, we limit the universality of the epochè. (If we did not) no province would be left for unmodified judgments, to say nothing of a province for science."10

The epoche must stop at the precise point where it meets the "province" that the phenomenologist wants to submit to careful scrutiny; namely the domain to which the objects of ordinary perception and ordinary action are reduced. This "province" to which everything else is reduced should not be put out of play in turn, as this would hinder attempts to turn it into a proper theme for a new kind of (phenomenological) knowledge.

These two steps of the phenomenological practice, the epochè that prepares reduction, and the reduction itself, are carefully distinguished in many texts of Husserl. Just consider these sentences: "I must put (objective nature) out of action, in order to achieve a reduction to the pure psychic realm"; "I must reduce the objective experience to its purely subjective being. For this, I have to submit the objective world to the epochè." 11 Here, the epoche is defined as the preliminary suspension whose finality is some sort of phenomenological reduction.

But this implies that the point at which the epoche is stopped can vary according to the type and depth of the phenomenological inquiry one wishes to perform. This gives rise to various reductions, at various levels of our psychic life. As Husserl stated, "(the) operation (of epochè) will be divided into different steps of 'putting out of action', 'parenthesizing'; therefore, our method will assume the characteristic of a step-by-step reduction. For this reason, we shall, on most occasions, speak of phenomenological reductions." 12 In other terms, the variety of points at which the epochè can be stopped define several steps of the reduction, several reductions.

\section{Lifeworld reduction and transcendental reduction}

In his Crisis of the European Sciences, Husserl individualized at least two steps in this process (although there are many others): (1) the lifeworld reduction, and (2) the transcendental reduction.

To perform the life-world reduction, one suspends any belief in the abstract theoretical entities of the natural sciences, and sticks to the concrete objects of everyday speech and manipulations. Now, what is the precise border between the life-world and the domain of science?

\footnotetext{
${ }^{10}$ Husserl (2016, §32)

${ }^{11}$ Husserl (2007)

${ }^{12}$ Husserl (2016, §33)
} 
Or, in Bas Van Fraassen's terms, what is the border between the observable and the unobservable? Husserl starts with saying that the lifeworld is identical with the standard furniture of ordinary life, seen in a purely prescientific light ${ }^{13}$. But he soon extends it to the furniture of scientific laboratories. Once the lifeworld reduction is performed, one no longer speaks in terms of electrons, quantum fields, etc. but rather in terms of heated metals, reflective cavities, detectors, etc. The scientific instruments and the vocabulary of experimental activity are retained on the side of the lifeworld, and therefore taken at face value, whereas any object allegedly referred to by theoretical symbols falls under the epoche. "For a physicist, Husserl writes, the lifeworld is the world in which he sees his measuring instruments, he listens the beats from his chronometers, he evaluates the magnitudes he observed, and so on." 14

This layer of the phenomenological reduction, the lifeworld reduction extended to laboratory life, is incomplete. For it only leads us to the domain of our collective conventions; it leads us to the set of items and situations one can easily communicate about, be they inside or outside the laboratory. But such lifeworld reduction has a high methodological value for science. It proves easy to apply to physics, and especially to quantum physics, since it just documents the shift between the attitude of an abstract theoretician who tends to grant a high ontological value to her formal entities, and the attitude of a downto-earth physicist who is permanently aware that experiments are the soil on which any theoretical claim relies. At any rate, the lifeworld reduction is exactly the kind of reduction that was documented in Bohr's and Heisenberg's works: withdrawal from pictures inspired from previous theories, and adhesion to the visible objects or events in labs, described in terms that are intersubjectively communicable.

At first sight, the alternative reduction, i.e. the transcendental reduction, looks completely irrelevant to physics. Performing the transcendental reduction is a highly demanding task of digging below the level of everyday and laboratory conventions. Here, one suspends not only the explicit belief in theoretical entities, but also the implicit beliefs that are conveyed by ordinary language, and crystallized in the perception of ordinary objects. Once this has been done, what is left is only the flux of lived experience, with both its perceptive and intellectual aspects. The task of a Husserlian phenomenologist is then to examine how our ordinary conceptions of the world and the standard

\footnotetext{
${ }^{13}$ Husserl (1976, p. 167)
}

${ }^{14}$ Husserl (1976, p. 139) 
entities of ordinary life are "constituted" out of this ground; just in the same way as one may inquire into whether it is possible to "constitute" the theoretical entities of science out of the body of evidence gathered at the mesoscopic scale of the laboratory.

But the challenge is even more daunting in the case of the transcendental reduction than in the case of the lifeworld reduction. For, when the transcendental reduction is performed, even the standard conditions for mutual understanding and intersubjective agreement about the enduring things that can be shown and manipulated, are suspended ${ }^{15}$.

Early signs of the need for transcendental deduction: experience, consciousness and quantum mechanics

Despite this challenge, signs that the transcendental reduction might be required by quantum physics, beyond the life-world reduction, are visible in various texts of its creators, and in some formulations of its so-called "paradoxes".

Bohr thus often referred to human experience, not only to pointer readings. That this (allegedly idealist) aspect of Bohr's thought has been played down in standard texts of the "Copenhagen school", and finally overshadowed in his later writings, might be due to the pressure of Soviet physicists who had to pay tribute to dialectic materialism ${ }^{16}$. But these historical circumstances cannot hide entirely the importance Bohr ascribed to human lived experience. Just read the following sentences: "When speaking of a conceptual framework, we refer merely to the unambiguous logical representation of relations between experiences" 17 ; "Science [aims] at the development of general methods for ordering common human experience." 18 It is true that "common human experience" can be conveniently expressed in terms of nouns and predicates referring to mesoscopic objects, and thereby neglected in favor of the familiar life-world. But at the end of the day, the benchmark of physics is to be found within single lived experiences in which the expectations derived from theoretical calculations are confronted with perceived pointer readings. Lived experience is the terminus a quo and the terminus ad quem of physics. In many cases this

\footnotetext{
15 Findlay (1948)

${ }^{16}$ Whitaker (1996, p. 166)

${ }^{17}$ Bohr (1987, p. 68)

${ }^{18}$ Bohr (1987, p. 80)
} 
is almost too trivial to be noticed; but in quantum physics, this might well have a crucial importance.

The measurement problem of quantum mechanics has been a revelator of this non-trivial role of lived experience. But the way this role has been expressed is usually flawed, not to say fanciful, because it relies on a disputable set of ontological presuppositions: (1) that conscious experience is either something or a property of something; and (2) that the physical systems and processes postulated by physics are things that exist out there, independently of consciousness. The standard way of introducing conscious experience in the measurement problem indeed implies a form of dualism. It implies there are two items, physical systems plus states on the one side, and conscious experiences on the other side. Accordingly, conscious experiences are ascribed the task of suddenly modifying the state of physical systems; of suddenly "collapsing" these "states" from superpositions to sharp values ${ }^{19}$. And the reason why conscious experiences are granted this uncanny ability is that they do not belong to the set of quantum objects whose "state" is liable to superposition; or, may be, that their nature is non-physical altogether.

\section{Transcendental philosophy at work in quantum physics: Von Neumann and Everett}

Apart from this dualistic caricature, some more nuanced (and phenomenology-compatible) approaches of the role of lived experience have been formulated from the very beginning of the history of the measurement problem. Unfortunately, they have been discarded too quickly because, to philosophically unsophisticated minds, they look like varieties of dualism. Let me give two examples.

Von Neumann's formulation of the measurement problem of quantum mechanics is paradigmatic. It is usually believed that Von Neumann was the first thinker to introduce the strange idea that consciousness is able to modify physical states by collapsing them from superpositions to sharp values. But in fact, his position was much more subtle, and mostly in tune with phenomenology. His key sentence is the following : "No matter how far we calculate - to the mercury vessel, to the scale of the thermometer, to the retina, or into the brain, at some time we must say: and this is perceived by the observer." ${ }^{20}$ According to von Neumann, the measurement problem cannot be solved by just

\footnotetext{
19 e.g. Stapp (2007)

${ }^{20}$ Von Neumann (1955)
} 
evoking some physical event that occurs in the brain of the observer at the end of a measuring interaction. For such event would remain "inside the (quantum) calculation" and would therefore do nothing to break the chain of entanglements and superpositions. But von Neumann does not make use of some non-physical entity either. He does not claim that there exists some ghostly "thing" or "property" called "consciousness" that is able to magically collapse physical states due to its non-physical nature. What he mentions is only a change in the level of description, that accounts for the difference between a superposition and a sharp eigenstate. From a neutral mode of description, one switches to a situated mode of description. The view from nowhere of a theoretician is replaced with a view from somewhere (the view of someone engaged in the ongoing measuring process, and able to witness its outcome). Someone who adopts the view from nowhere is bound to use the superposition of states, while someone who adopts the view from somewhere is led to use the sharp eigenstate instead, for the same sake of predicting the outcomes of future measurements. No miracle occurs here, but only a change in one's self-ascribed epistemological status: from anonymous predictor to specific observer, from a neutral stance to a situated view. Both state vectors (superposed and sharp) can be used alternatively by one and the same person, according to her needs: either providing a weighted list of possible experiences available to anyone, or indicating the actual experience of someone.

This non-substantialist construal of observers and their consciousness is confirmed by von Neumann's use of the quasiHusserlian expression "abstract ego" (Husserl would have written "transcendental ego"). According to von Neumann, the divide between the observer and the observed system can be moved back further and further until nothing (not even a brain, not even a ghostly soul) is left on the observer's side. It can be moved until the observer is represented only by her "abstract ego", namely by a pure knower unknowable to itself, whereas all the rest is treated as a global (quantum) system. This procedure clearly precludes any reification of the observer's residue. What is left on the observer's side is no particular thing, even though it is not nothing. It is a pure, fleeting, present experience of perceiving.

Something similar can be found in Everett's interpretation of quantum mechanics. Everett's crucial move consists not so much in adding one more element to the measurement chain (say a physical observer or, may be, a recording robot), as in appending a new symbol to its state. The new symbol is a "memory bracket", that contains a list of memories of the measurement outcomes which have been observed 
and recorded in the past by an observer. In Everett's formulas, there are as many memory brackets as there are terms in the entangled superposition of the global state vector of the measurement chain. So, each memory bracket is supposed to hold not in the absolute, but only relative to the corresponding term. In the many-world re-interpretation of Everett's interpretation, this relativity is made even more concrete, since it is claimed that each memory bracket holds within the world that corresponds to this term.

However, the mere addition of a symbol to each term of the superposition is not sufficient by itself to solve the measurement problem, since no collapse is triggered by it. What really does the trick is the situated meaning ascribed to the symbol "memory bracket". The measurement problem is arguably solved when one endorses the following kind of statement:

"From my observer's point of view, in my experience, it appears that a sharp outcome has been obtained, even though from the standpoint

of distanciated predictors, the initial superposed state is still valid".

So, the solution of the measurement problem here arises from full awareness that one occupies an idiosyncratic situation, and that this situation self-manifests in one's own lived experience of some particular measurement outcome. Here again (as in von Neumann) consciousness does nothing to the physical world. Instead, the so-called "events of the physical world" are reinterpreted as a handy way to express the common focus of the expectations and observations of situated agents endowed with conscious experiences.

\section{The transcendental reduction of QBism}

Yet, the most consistent phenomenological approach of quantum mechanics is presumably QBism ${ }^{21}$. QBism is an acronym for "Quantum Bayesianism". According to QBism, "state" vectors are just probabilistic valuations, in a Bayesian sense. They are not statements about what is the case, but statements about what each agent can reasonably expect to be the case. Ultimately, they are mere expressions of subjective guesses; they express subjective agent's willingness to place bets about each outcome. Hence the alternative expression "Quantum Bettabilitarianism".

A feature that makes QBism definitely akin to phenomenology is that it adopts a deliberately first-person standpoint (be it first-person singular or first-person plural). The project of both phenomenology and

${ }^{21}$ Fuchs et al. (2014); Von Baeyer (2016) 
QBism is to reconstruct the so-called objective knowledge, by starting afresh from the first-person standpoint of knowers or agents. Just as good phenomenologists, QBist thinkers suspend judgment about a presumably external domain of objects: they perform a strong variety of Husserl's epochè. Indeed, in QBism, the symbols of quantum theories are no longer supposed to refer to objects, nor are they supposed to denote predicates of objects. Then, since the attention of QBists is no longer absorbed by claims about objects, it is reflectively redirected towards the epistemic function and the practical use of the symbols of quantum mechanics. QBists focus on the fact that the symbols of quantum mechanics are primarily used by agents to assign probabilistic weights to (agent's future experiences of) various outcomes of experiments, so as to make consistent bets about them.

This reflective move that comes after the epochè, this focusing on the agent's activity of anticipation and probability ascription, is clearly akin to Husserl's phenomenological reduction. But which kind of reduction? The very way we have formulated the QBist's reflective move suggests an answer. Probabilities bear on future experiences (not just on future pointer positions), and anticipation is a mental activity (not just a set of symbols written on a blackboard). The QBist reduction is thus, unambiguously, a form of transcendental reduction.

Chris Fuchs himself clearly states that his reduction cannot be restricted to the kind of lifeworld reduction that Bohr advocated. As alluded to out earlier, he insists that his starting point and unique theme is the lived experience of agents. According to QBism, the quantum "state" has no direct representational bearing on physical processes; it is a symbolic tool within "a calculus for gambling on each agent's own experience." ${ }^{22}$ This confirms that QBism arises from an act of "transcendental reduction" in Husserl's sense.

Moreover, this act of transcendental reduction motivates a highly non-standard ontology. In Chris Fuchs' terms, "I do think we have a kind of direct evidence of 'the real'. It is in the very notion of experience itself." ${ }^{23}$ A plausible interpretation of this latter sentence is that only in experience do we know directly "reality". Indeed, in experience we know reality by acquaintance, rather than by distanciating ourselves from it. What may look strange from a naturalistic standpoint is that such acquainted reality is neither a "reality out there" nor some "inner reality" but just a present overarching reality. Reality is a continuum we

\footnotetext{
${ }^{22}$ Fuchs (2010)

${ }^{23}$ Fuchs (2017)
} 
partake of, not something we contemplate from without or something we encapsulate within.

This idea is in tune with the very spirit of phenomenology, and especially with phenomenological ontologies. The typical claim of phenomenological ontologies is that Being is strictly coextensive to Appearing. The list of quotations of phenomenologists who support it, is almost inexhaustible. Accordingly, this premise has been transformed into a slogan by the main lineage of contemporary French phenomenologists, from Michel Henry to Jean-Luc Marion and Renaud Barbaras: "autant d'apparaître, autant d'être." ${ }^{24}$ Such slogan is usually translated into English as follows: "something is inasmuch as it appears". But the latter translation is ambiguous, because it sounds as if there were something whose appearance is the criterion of Being. The true premise of phenomenology differs considerably from this interpretation. Indeed, according to it, being is nothing above and beyond appearing, nothing above and beyond experience. It is true that there is also a phenomenological sense of the transcendence of things with respect to their appearing; but this feeling of transcendence is selfgenerated by the very structure of appearance, and it is therefore aptly called "transcendence in immanence". As Eugen Fink wrote unambiguously, "(Phenomenology) simply claims that being is identical with the phenomenon." 25 Similarly, according to Heidegger, "Being means appearing"; "Appearing does not mean something derivative, which from time to time meets up with Being. Being essentially unfolds as appearing." 26

\section{Back to the lifeworld reduction (for the moment)}

Of course, granting such privilege to transcendental reduction over the life-world reduction in the domain of physics, raises difficult issues. Indeed, after the epochè has been pushed to a level that enables one to perform the transcendental reduction, the basic entities that are usually presupposed by the discourse of physicists are suspended, they are "put out of action"; and, therefore, the conditions for intersubjective agreement between physicists are no longer available. These object-like conditions for intersubjective agreement must then be stated explicitly. But how can this be done?

\footnotetext{
${ }^{24}$ Henry (1991)

${ }^{25}$ Fink (1994, p. 120)

${ }^{26}$ Heidegger (1980, p. 109)
} 
David Mermin proposed to proceed as follows: "Although I cannot enter your mind to experience your own private perceptions, you can affect my perceptions through language. When I converse with you or read your books and articles in Nature, I plausibly conclude that you are a perceiving being rather like myself, and infer features of your experience." 27 This is fine. But, here again, we must not forget that our mutual understanding through language is based on the shared assumption of the ordinary objects of our direct perception, denomination, and predication. In other terms, it is based on the level of life-world reduction, not on the level of transcendental reduction. And when we try to evoke the level of "pure experience", it is by way of an essentially allegoric language that uses comparisons with the domain of ordinary perceptions and ordinary life. So, even though QBism is phenomenologically right to claim that the de jure basis of scientific knowledge is personal lived experience and verbal communication between subjects of experience, it should also recognize that the de facto basis of quantum physics is Bohr's classicallike domain of ordinary objects and instruments. This would avoid several difficulties in the discourse of QBism.

Indeed, whereas one can say that living always involves anticipating what may come next in experience, probability assignments do not reduce to anticipations of this elementary kind. Unlike perceptive anticipations, probability assignments are formalized anticipations, and these formalized anticipations bear on highly elaborated types (rather than tokens) of experiences. Such types of experiences are most conveniently expressed in terms of classical-like predicates of instruments. For example, one would say: "I have observed that the Stern-Gerlach pointer was on 'up' position"; or "I ascribe probability $1 / 2$ to the 'up' pointer-reading in this Stern-Gerlach experiment". "I observe" and "I ascribe" concern personal experiences and choices, but what comes after the words "that" and "to" is bound to use ordinary language and classical-like presuppositions. For this is the only way to reach the domain of what is common to all agents.

Moreover, when one is asked to explain the structure of the quantum probabilistic predictions, one must go beyond the purely subjectivistic option of QBism. Let me take an example. Why do we predict interference patterns on a screen after a double-slit device? The standard explanation is that there must be waves "out there", that selfinterfere along the two paths made available by the double-slit device; and that these real waves are represented by the wave-functions of

${ }^{27}$ Mermin (2014) 
quantum mechanics. But there is an alternative, non-realist explanation. This alternative explanation is that it can be shown that wave-like appearances are bound to be forecasted, whenever a probabilistic prediction bears on contextual phenomena ${ }^{28}$. Here, the gist of the new explanation is neither to be found in "outer reality" nor in "the inner recesses of subjects", but somewhere in between. It relies on the interface between the outer and inner, on the fact that phenomena are relative to what triggers them (the experimental context), on the creative activity of agents who use specific types of devices to explore their cryptic environment. It is then clear that no personal arbitrariness is involved in the quantum form of probabilistic predictions; no "subjectivity" in the ordinary and narrow sense of something purely private, of something that only holds for someone. To formulate predictions about phenomena, a physicist follows rules (or norms) that take into account the whole experimental pattern. And this experimental pattern is described (as Bohr would have it) in ordinary language plus classical terminology. To recapitulate, the privilege given to transcendental reduction in QBism cannot entirely bypass the relevance of the more moderate life-world reduction for explanation and justification.

\section{On the necessity of practicing the transcendental reduction}

But then, why does QBism pays so little attention to the life-world reduction and rather stick to the transcendental reduction? Is this latter kind of reduction really indispensable for the clarification of quantum mechanics? I definitely think so. There are several reasons in quantum mechanics for performing the transcendental reduction beyond the lifeworld reduction. And, taken together, these reasons appear extremely compelling.

The first reason is Wigner's friend so-called "paradox", which appears as a mystery in a realist framework, which remains a mystery after one has performed the life-world reduction, but which is immediately dissolved once the transcendental reduction has been performed in the wake of QBism.

To understand the QBists' reading of Wigner's friend thought experiment, we must start with examining once again the QBist view of quantum "state vectors". As we now realize, in QBism, the quantum "state" has no direct bearing on physical processes; it is a symbol for

${ }^{28}$ Destouches-Février (1951) 
gambling on agent's experiences ${ }^{29}$. This entails that (i) the reduction of the "state" represents no "physical" process, and (ii) in the process of quantum "gambling", lived experience plays the role of the most universal presupposition, rather than being taken for some airy additional ingredient grafted onto a world fundamentally made of physical objects. As a consequence, in QBism, there is nothing like an "objective reduction of the physical state"; there is only a change in expectations (a change in the dispositions to bet) that takes into account previous experiences of measurement outcomes.

So, let's acknowledge that, in QBism, quantum symbols, and especially state vectors, bear exclusively on experiences. Let's aknowledge that they bear on experiences' being expected or being felt, on their being conceived as possible or their being sensed as real, and nothing else. It is then trivial to understand why Wigner (who is outside the laboratory) does not use the same state vector as his friend (who is inside the laboratory). This is due to a difference between the informational bases on which those two researchers endowed with lived experience rely for elaborating their optimal bets about future experiences. "One statement refers to the friend's potential experiences, and one refers to Wigner's own" ${ }^{30}$. Since nothing else than conscious experience is involved in the symbols of quantum physics, no action of conscious experience on something else must be called upon to account for sudden changes in these symbols. What accounts for such changes is just that conscious beings modify their dispositions to bet, according to the information they have retrieved in their conscious minds.

If we had stopped the epochè at some upper level, say by performing the life-world reduction without performing the transcendental reduction, Wigner's friend thought experiment would have been much more difficult to account for. In this case, we would have had to find a reason, in the mesoscopic domain of experimental devices and laboratory activities, why only one of the two state vectors is valid. Either Wigner's superposed state vector holds, or his friend's sharp state vector holds, but not both of them. The standard, Copenhagen-like, explanation of this difference in validity between the two state vectors, is that a series of irreversible events occurred earlier in the experimental apparatus, yielding the stabilization of a pointer reading which thereby indicate a sharp value. In such case, the sharp state ascribed by Wigner's friend to the measurement chain (that includes the "physical system" and the apparatus) reflects the real state of the mesoscopic

\footnotetext{
${ }^{29}$ Fuchs (2010)

${ }^{30}$ Ibid.
} 
furniture of the laboratory. This particular state is deemed to be real because it expresses an intersubjectively valid configuration; a configuration that can be communicated by and to human observers using ordinary language plus classical concepts.

By contrast, the superposed state ascribed by Wigner to the same measurement chain that includes his friend is said to reflect Wigner's ignorance of the real state of what is inside the laboratory. But if this is so, Wigner's superposed state should bear the mark of the said ignorance. It should become a statistical mixture, rather than remaining a superposition.

\section{Decoherence and the persistent relevance of the transcendental reduction}

The physicists' hope was that the transition from a full-blown superposition to a statistical mixture could be derived from the quantum formalism, together with some thermodynamical considerations. And, to a certain extent, they have achieved this successfully by way of the decoherence procedure; but to a limited extent only. Decoherence was meant to bridge the gap between the quantum domain of superpositions and the classical domain of sharp properties, by way of "environmentinduced superselection" 31 . The principle of this solution consists in showing that the phase coherences of the state vector (or the density operator) of an apparatus correlated to a micro-system are rapidly diluted in its vast environment. Indeed, the virtually complete disappearance of the interference terms is equivalent to a superselection rule by which one only retains the eigenstates of a given observable.

But beware: such superselection is always incomplete. For the interference terms do not entirely disappear; they just become negligible. And these interference terms can even recur in the very long run. Decoherence then yields an "improper" statistical mixture, rather than a "proper" one ${ }^{32}$. State vector evolution does not "properly" describe the advent of a set of sharp properties of objects, that would be typical of the lifeworld's "natural" ontology. Even after decoherence, state vectors persistently denote in principle (if not in practice) the suspension of any determination, and the relativity of present determinations to an act of cognizance that will manifest in future experience. Even after decoherence, quantum symbols bear the mark of their limited status: they do not describe present intrinsic

\footnotetext{
${ }^{31}$ Zurek (2003)

${ }^{32}$ D’Espagnat (2003)
} 
determinations; they are just meant to predict (probabilistically) future events suspended to future experimental activities and to future acts of cognizance.

It then turns out that any discourse bearing on present ordinary properties and objects of our everyday environment, which is typical of the lifeworld reduction, is bound to remain an approximation in the quantum domain. And that the reference to future lived experiences, to future conscious acts of cognizance, cannot be completely avoided. The life-world reduction (to the laboratory furniture) must be complemented by a transcendental reduction (to pure awareness of laboratory activities and outcomes), if we are to make sense of the incompleteness of decoherence.

Let me recapitulate what I take to be the teaching of (the limits of) decoherence. In the quantum domain, there is no certainty inscribed "out there" in the heart of things, of which we would simply be aware or ignorant. The only true certainties are lying within our experience; they arise in our experience either ahead of events, or on the occasion of our being acquainted with them. The first form of certainty (ahead of events) is expressed by the probability 1 we now ascribe to some future measurement result, meaning that we are presently ready to bet our life on it. And the second form of certainty (on the occasion of our acquaintance with events) bears on what we are living in the present moment (be it in a laboratory or in everyday life).

No delegation of the certainty of experience to the objects of experience has ever proved unshakable. This is precisely what Husserl pointed out while he was practicing the transcendental reduction. Whereas consciousness is the "realm of absolute being" 33 , he wrote, the objects of our everyday environment only "claim being" 34 ; for, whereas consciousness is just given at once, the objects of our everyday environment are presented incompletely through partial aspects, profiles, or "adumbrations".

\section{Doubts about "objective facts": an incentive to perform the transcendental reduction}

We have just documented two kinds of certainties lying within our experiences: certainty about the present reasons of our guesses bearing on the future, and certainty about the intuitive presence of contemporary events. But what about our past? Are we not more certain

\footnotetext{
${ }^{33}$ Husserl $(2016, \S 76)$

${ }^{34}$ Husserl (1995, First meditation §8)
} 
of our past than of anything else? Isn't the word "fact", derived from the latin past participle "factum", a perfect expression of this kind of certainty? Let's inquire a little further. How can we be so sure of our past? First and foremost, there is our certainty of memories. Memories are experiences; they are often vivid present experiences of which we are just as certain as of any other present lived experience. However, the content of our memory is by no means as certain as the memory itself; not only because there are "false memories", but also because there are wrong interpretations. So, our convictions about the past must also rely on another type of certainty.

Strangely enough, our convictions about the past rely on a reasonable amount of anticipation of our future. On the basis of our memories, we assume that in the future we'll find a bundle of traces, recordings, and reports that will confirm the descriptive content of such memories. We would sometimes bet our lives that further recordings will be consistent with our present memories. But, of course, this kind of confirmation is not always obtained. Our expectations about recordings, traces, and reports can be disappointed. And from then on, the past is no longer what it was.

Now, what about the opposite case where our expectations about recordings and newly rediscovered archives are indeed fulfilled? In the classical paradigm, this confirmation would be sufficient to bring back absolute certainty about the past. For, in the classical paradigm, even if we are not entirely confident about the descriptive content of our memories, we can at least be absolutely sure of the properties and objects of the life-world; and these include recordings, traces and reports. In the classical paradigm, certainty is inscribed "out there" in the heart of things, and it is therefore also inscribed "out there" in the traces borne by things.

In the quantum paradigm, however, the situation is quite different. The principle of quantum physics is prediction, not description. And not preduction of intrinsically occurring facts, but prediction of ever unaccomplished events, suspended to their joint creation by an experimental act. As Bernard d'Espagnat noticed long ago, "Within standard quantum mechanics, (there are) no 'really existing' facts" 35 . In the quantum paradigm, any claim about facts is suspended to a future experimental act. And even when facts are recorded, memorized, written down, etc. they are still suspended to future acts of deciphering records, retrieving memories, reading written marks etc. Decoherence,

${ }^{35}$ B. d'Espagnat, "Towards an empirical separable reality?", Foundations of Physics, 20, 11471172,1990 
that was aimed at restoring the classical certainty inscribed "out there", has shown its in principle loophole in this respect. Last but not least, there is now a fresh wave of reflections, thought experiments (of Wigner's type) ${ }^{36}$, and proposed laboratory experiments ${ }^{37}$, whose impressive teaching is that, to make sense of quantum predictions one must accept that even "facts are relative" 38 .

The standard certainties of the life-world being thus no longer available, we must content ourselves with the bare certainty of transcendental consciousness. Once again, it looks like it is quantum physics itself that does not allow us to content ourselves with the lifeworld reduction, and rather invites us to perform the transcendental reduction.

\section{Non-locality or transcendental reduction?}

Now, is there a situation, in quantum physics, where performing the transcendental reduction is not only an invitation but almost an obligation? Is there a situation, in quantum physics, where we have no other reasonable option than considering that "facts", far from being absolute, far from having occurred by themselves in the past, are always suspended to future acts of bringing "them" out? I think there is such a situation. It is the dubious but widespread opinion according to which quantum mechanics implies "non-locality".

Why do I claim that the quantum non-locality is only an opinion? For the simple reason that Bell's theorem, and the violation of Bell's inequalities by quantum predictions, can be interpreted in at least two ways, and that one of these ways does not involve anything like nonlocality. As it is well known ${ }^{39}$, Bell's inequalities (and every further inequalities of this kind) are derivable from two assumptions: (i) realism about micro-properties (or macro-properties) ${ }^{40}$, and (ii) locality of these properties. In this case, violation of such inequalities by quantum predictions and by experimental results fitting with quantum predictions, can be accounted for in two ways. Either there exist non-

\footnotetext{
${ }^{36}$ Brukner (2018)

${ }^{37}$ Bong et al. (2020)

${ }^{38}$ Brukner (2020)

${ }^{39}$ D’Espagnat (1975)

${ }^{40}$ Some authors have challenged the necessity of the first assumption. See Laudisa (2019). But even though realism about microproperties is not indispensible to derive Bell's inequalities, a weaker form of realism (realism about laboratory "facts") is needed. See Bell (1981). The recent burst of challenges of the concept of "intrinsic" fact is a good confirmation that "fact-realism" is indeed a problematic assumption despite its looking innocently common sense.
} 
local influences between micro-properties, or there is no such thing as an "intrinsically real" property.

It is only because the second option, namely property anti-realism, does not look attractive to many physicists, that the first option, namely non-locality, became so popular. Nevertheless, almost everything points towards the opposite direction. Bohr's insistence on the contextuality of micro-properties and representations, clearly favors micro-property anti-realism. Moreover, it has been shown that the putative non-locality of quantum mechanics is purely formal; that nonlocality is just a projection in ontological terms of the symbolic form of entangled states. Indeed, the no-signalling theorem ${ }^{41}$ has demonstrated that the so-called non-locality has no observable consequence, that it implies no faster-than-light transfers of information. Not even the socalled "quantum teleportation" can overcome this limitation, since it requires a classical (slower than light) canal of information.

Yet, one could reply, aren't quantum entangled states describing strict correlations between space-like separated events; and can't these latter correlations be detected experimentally? Aren't then such detected correlations sufficient evidence that quantum mechanics imply non-local effects? For, isn't non-local causal influence the only plausible explanation of these correlations?

Beware at this point: the very initial claim that correlations between space-like separated events are described by quantum mechanics can only arise from a descriptive, and therefore "realist", construal of quantum states; and therefore, deriving the "reality" of correlations from this argument is a petitio principii. As for the further claim that correlations between space-like separated events are detected experimentally, it misses the obvious circumstance that such correlations can be brought out only much later, when the signals conveying the information about correlated events are no longer spacelike separated! Remember John Wheeler's celebrated warning, that no phenomenon is a phenomenon until it is an observed phenomenon. Here, we must add: no correlation is a correlation until it is an observed correlation $^{42}$. Quantum entangled states predict that a correlation will be observed in the future with a high probability; they do not describe or express the correlation, since the latter does not yet exist in any concrete sense of the verb "to exist".

This is the reason why QBists bluntly deny the so-called "quantum non-locality". A single sentence suffices for them to blow out a whole

\footnotetext{
${ }^{41}$ Peres \& Terno (2004)

${ }^{42}$ Bitbol (1983, 2015); Smerlak \& Rovelli (2007)
} 
tradition of wondering about the non-local "magics" of the quantum world: "There is no nonlocality in quantum theory; there are only some nonlocal interpretations of quantum mechanics". QBism clearly does not belong to the subset of non-local interpretations, since it does not uphold anything like a "realist" view of quantum states. Since agents use quantum states to no other purpose than predicting correlations "between the manifold aspects of [their] experience", and since agent's lived experiences "cannot be space-like separated", there is no such thing as a correlation between space-like separated events. To insist, "Quantum correlations, by their very nature, refer only to time-like separated events: the acquisition of experiences by any single agent" 43 .

Here, the QBist option of performing a transcendental reduction straightaway, and avoiding the intermediate step of the life-world reduction, immediately manifests a momentous consequence. This consequence is a dissolution of all the conundrums associated with the putative non-locality of quantum mechanics. Indeed, from this standpoint, non-locality is just a fake descriptive projection of a mathematical property of the predictive symbol of quantum physics (entanglement, or non-factorizability, of the state vector). That this indeed represents a fake inference is strongly suggested, once again, by the lack of any possibility of faster-than-light communication.

My conclusion is that nothing less than the most extreme transcendental reduction to the living present can allow us to dispel the joint enigmas of quantum correlations and quantum decoherence. Nothing less than the most extreme transcendental reduction to the living present can maintain a full conceptual coherence within the quantum paradigm. I then concur with QBists when they suggest that Bohr's life-world reduction is not sufficient to make full sense of the quantum paradigm.

\section{References}

Bell J.S. 1981. Bertlmann Socks and the nature of reality. Journal de Physique. suppl., 42, C2: 41-62

Bitbol M. 1983. An analysis of the Einstein-Podolsky-Rosen correlations in terms of events. Physics Letters 96A. 66-70

Bitbol M. 2015. La pratique des possibles. Paris: Hermann

Boehm R. 1965. Basic reflections on Husserl's phenomenological reduction. International Philosophical Quarterly 5: 183-202

${ }^{43}$ Fuchs et al. (2014) 
Bong K.-W., A. Utreiras-Alarcon, F. Ghafari, Y.C. Liang, N. Tischler, E.G. Cavalcanti, G.J. Pryde, \& H.M. Wiseman. 2020. A strong no-go theorem on the Wigner's friend paradox. Nature Physics. https://doi.org/10.1038/s41567-0200990-X

Brukner Č. 2018. A No-Go theorem for Observer-Independent Facts. Entropy. 20: 350

Brukner Č. 2020. Facts are relative. Nature Physics. https://doi.org/10.1038/s41567-020-0990-X

Bohr, N. 1987. Atomic Physics and Human Knowledge. New York: John Wiley

Destouches-Février P. 1951. La structure des théories physiques. Paris: Presses Universitaires de France

Espagnat B. d'. 1975. Use of inequalities for the experimental test of a general conception of the foundations of microphysics. Physical Review D. 11: 14241435

Espagnat B. d'. 2003. Veiled Reality, Boulder: Westview Press

Findlay J.N. 1948. Recommendations regarding the language of introspection. Philosophy and Phenomenological Research 9: 212-236.

Fink E. 1994. Proximité et distance. Grenoble: Jérôme Millon

Fuchs C.A. 2010. QBism, the perimeter of quantum bayesianism. arXiv: 1003.5209 [quant-ph]

Fuchs C.A., N.D. Mermin, R. Schack. 2014. An Introduction to QBism with an Application to the Locality of Quantum Mechanics. American Journal of Physics 82: $749-754$

Fuchs C.A. 2017. On participatory realism. In Information and Interaction, eds. Ian Durham and Dean Rickles. Berlin: Springer

Heidegger M. 1980. Introduction à la métaphysique. Paris: Gallimard

Heisenberg, W. 1925. Über quantentheoretische Umdeutung kinematischer und mechanischer Beziehungen. Zeitschrift für Physik 33: 879-893

Henry M. 1991. Quatre principes de la phénoménologie. Revue de Métaphysique et de Morale. 96: 3-26

Husserl, E. 1964. The Idea of Phenomenology. Dordrecht: Kluwer

Husserl, E. 1976. La crise des sciences européennes. Paris: Gallimard

Husserl, E. 1995. Cartesian Meditations: An Introduction to Phenomenology. Dordrecht: Kluwer

Husserl, E. 2007. De la réduction phénoménologique (1926-1935), Grenoble: Jérôme Millon

Husserl, E. 2016. Ideas for a pure Phenomenology and Phenomenological Philosophy. Indianapolis: Hackett 
Laudisa L. 2019. Counterfactual Reasoning, Realism and Quantum Mechanics: Much Ado About Nothing? Erkenntnis 84: 1103-1118

Mermin D. 2014. QBism puts the scientist back into science. Nature 507: 421-423

Patočka J. 2002. Qu'est-ce que la phénoménologie? Grenoble: Jérôme Millon

Peres A. \& D.R. Terno 2004. Quantum information and relativity theory. Review of Modern Physics: 93-123

Perniola, M. 2011. The expanded epochè. Iris 3: 157-170

Smerlak M. \& C. Rovelli 2007. Relational EPR. Foundations of Physics. 37: 427445

Stapp H. 2007. Mindful Universe: Quantum Mechanics and the Participating Observer. Berlin : Springer

Von Baeyer H. 2016. QBism, the Future of Quantum Physics. Cambridge: Harvard University Press

Von Neumann J. 1955. Mathematical Foundations of Quantum Mechanics, Princeton: Princeton University Press

Whitaker A. 1996. Einstein, Bohr, and the Quantum Dilemma. Cambridge: Cambridge University Press

Zurek W.H. 2003. Decoherence, einselection, and the quantum origins of the classical. Review of Modern Physics 75: 715-775 